\title{
Multilinguales
}

\section{L'ambivalence spatiale comme symbolique de l'ambivalence identitaire dans Histoire de ma vie de Fadhma Aïth Mansour Amrouche?}

The spatial ambivalence as symbolic of identity ambivalence in History of my life of Fadhma Aith Mansour Amrouche?

$$
\text { منصور عمروجية الفضاء المكاني سبب ازدواجية الهوية في قصة حياتي لفاطمة أث }
$$

\section{Kamel Medjdoub}

\section{CpenEdition}

Édition électronique

URL : http://journals.openedition.org/multilinguales/523

DOI : $10.4000 /$ multilinguales.523

ISSN : 2335-1853

\section{Éditeur}

Université Abderrahmane Mira - Bejaia

\section{Référence électronique}

Kamel Medjdoub, «L'ambivalence spatiale comme symbolique de l'ambivalence identitaire dans Histoire de ma vie de Fadhma Aith Mansour Amrouche ? ", Multilinguales [En ligne], 8| 2017, mis en ligne le 01 juin 2017, consulté le 17 septembre 2019. URL : http://journals.openedition.org/ multilinguales/523; DOI : 10.4000/multilinguales.523

Ce document a été généré automatiquement le 17 septembre 2019.

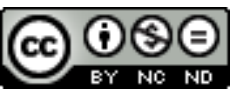

Multilinguales est mise à disposition selon les termes de la Licence Creative Commons Attribution Pas d'Utilisation Commerciale - Pas de Modification 4.0 International 


\title{
L'ambivalence spatiale comme symbolique de l'ambivalence identitaire dans Histoire de ma vie de Fadhma Aith Mansour Amrouche?
}

\author{
The spatial ambivalence as symbolic of identity ambivalence in History of my \\ life of Fadhma Aith Mansour Amrouche? \\ ازدواجية الفضاء المكاني سبب ازدواجية الهوية في قصة حياتي لفاطمة أث

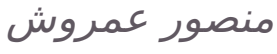

\section{Kamel Medjdoub}

1 L'écriture de l'errance entretient un lien avec les frontières, en tant que limites spatiales qu'elle abolit. Ce lien étroit transparaît dans Histoire de ma vie de Fadhma Aïth Mansour Amrouche (2009), où l'espace est autant physique que mental.

2 Histoire de ma vie est un récit autobiographique où le personnage-narrateur-auteur est engagé sur le chemin de l'errance depuis son enfance, à la fin du XIXe siècle, jusqu'au crépuscule de sa vie, au milieu du XXe siècle. Son errance va au delà des frontières nationales et l'a condamnée non pas à un exil mais à des exils. Avant d'aller plus loin dans notre analyse, il nous faudra distinguer d'abord le double sens que revêt le mot exil.

3 Selon le dictionnaire Hachette (1999: 692), exil signifie « action d'expulser quelqu'un hors de sa patrie sans possibilité de retour ». Bien entendu, la narratrice n'a pas été bannie par une quelconque autorité administrative ou militaire. Elle a plutôt été "expulsée " par une conjoncture particulière. Elle a dû se séparer de sa patrie mais n'a pas été "sans possibilité de retour » à son pays d'origine. Son errance est marquée par des retours répétés au pays natal, "ou du moins par de fréquents allers et venues, ce qui conduit à nuancer le terme d'exil" (Bonnet, 1997 : 278). À se fier donc à ce premier sens, le seul véritable exil de la narratrice est celui qui, à la fin de son récit, lui a imposé un aller sans retour en France. 
4 Dans un deuxième sens, le mot exil signifie, selon toujours Hachette, "séjour obligé et pénible loin de ses proches, de ce à quoi l'on est attaché ». Dans ce cas, il nous semble juste de dire que Fadhma Aïth Mansour Amrouche est une exilée. Mieux, qu'elle a connu des exils du fait qu'elle a vécu plus d'un «séjour obligé» loin des siens. Et elle le dit: «[...] cette langue qui me fut un réconfort tout au long de mes exils " (Aïth Mansour Amrouche, 2009 : 208). S'agissant de la narratrice, il est donc question d'exils.

Omniprésent dans le récit, l'espace est intimement lié à tous ces déplacements. Errant "comme une âme en peine d'un endroit à l'autre» (48), Fadhma Amrouche renouvelle malgré elle les ruptures et les va-et-vient qui se répètent entre Tizi-Hibel, Taddert-ouFella, Aïth Maneguelleth, Ighil-Ali, Tunis, Nice et enfin Paris.

6 La relation est étroite entre cette écriture de l'errance et l'espace qui peut « se présenter comme un champ d'action ouvert ou au contraire comme une source d'oppression " (Paravy, 1999 : 18).

7 Florence Paravy relève que l'espace, qu'elle a étudié dans le roman africain francophone contemporain, "est avant tout un espace vécu, dont l'individu est le centre" (Paravy. : 06). On considérera de ce fait, qu'il est « fondamental pour l'homme de reconnaître l'espace qui l'environne, d'y trouver des repères qui donnent un sens à la place qu'il occupe" (Paravy : 07). L'individu tire profit de l'espace physique à travers ses « fonctions diverses: fonctions primaires de l'abri et du lieu nourricier, fonctions socio-économiques, idéologiques, etc.» (Paravy: 07). L'espace, en tant qu'aussi « architecture» et «topographie», n'est pas moins "un véritable objet sémiotique qui livre les secrets des fondements sous-jacents d'une société» (Paravy: 08).

8 L'espace est donc à lire, à comprendre et à ne pas dissocier des «oppositions fondamentales » qui impliquent l'individu. Ces oppositions peuvent paraître «purement spatiales» comme lorsqu'il s'agit de "haut vs bas ». Elles peuvent également paraître avec des « déterminations sociologiques, culturelles, religieuses ou idéologiques» (Paravy: 09), lorsqu'il s'agit par exemple d'espace " privé vs public » (Paravy : 09).

9 L'espace peut aussi donner lieu à des interprétations symboliques à travers « des images fondamentales, fondées sur le dynamisme des formes, (ouvert vs clos,...) ou le symbolisme du mouvement (statique vs dynamique,...) 》 (Paravy : 09)

10 Nous tenterons de relever certaines des «oppositions fondamentales " qui se manifestent dans Histoire de ma vie ainsi que leurs «interprétations symboliques». Pour ce faire, nous présenterons l'espace dans son ambivalence, et dans ses deux aspects physique et mental. Nous pensons que cette ambivalence est l'interprétation symbolique de l'ambivalence identitaire du personnage de la narratrice, déchirée entre deux cultures, kabyle et française.

11 Avant de pouvoir aboutir à cette conclusion, suivons d'abord les traces de Fadhma Aïth Mansour Amrouche pour comprendre son errance.

\section{De Tizi-Hibel à Paris : sur les traces de Fadhma}

Le récit de Histoire de ma vie commence dans un espace, se prolonge dans un autre et se termine dans un troisième, sur la rive nord de la Méditerranée.

13 La narratrice est née, d'une relation extraconjugale, en 1883, à Tizi-Hibel, un village kabyle, en Algérie, où a vécu sa mère, musulmane pratiquante. C'est dans cet espace 
qu'elle a vécu deux à trois ans avant que sa mère "un mercredi, jour de marché, [ne la] chargea sur son dos et [1]'emmena aux Ouadhias " (Aïth Mansour Amrouche, 2009 : 27) chez les Sœurs Blanches pour la mettre à l'abri des persécutions et de "la méchanceté des hommes» (27) qui voyaient en elle «l'enfant de la faute» (26). Nous sommes toujours sur les hauteurs de la Kabylie, la rupture n'est pas spatiale, mais cette première séparation de l'enfant d'avec le giron maternel a établi son tout premier contact avec les chrétiens avant que la mère ne reprenne sa petite fille qu'elle délivre de la maltraitance des Sœurs Blanches de l'orphelinat des Ouadhias : "quand ma mère vint le mercredi suivant, elle trouva encore les traces des coups sur tout mon corps" (28). Elle a dû revenir à son village natal mais qu'elle quittera ensuite pour, cette fois-ci, l'école des filles de Taddert-ou-Fella, toujours sur les hauteurs de la Kabylie. Du haut de ses quatre ans, en 1886, sa mère " consentit [donc] à se séparer » (30) d'elle encore une fois. Cette deuxième séparation durera plus longtemps que la première parce que "c'est seulement en $1890 \mathrm{ou}$ 91 que [Fadhma] revi[t] la maison de [sa] mère et [son] village » (36). Elle fréquentera cette école pendant dix ans et où elle recevra une instruction qui la distinguera de la majorité des femmes kabyles otages de la société patriarcale et conservatrice. À Taddert-ou-Fella, c'est un nouvel espace et de nouveaux repères qui se présentent mais desquels la petite Fadhma se séparera une première fois en 1895 après une première fermeture de l'école. Rouverte quelques mois plus tard, l'école ferme définitivement ses portes. La narratrice recharge alors sa malle et reprend le chemin du retour au village natal. La rupture est celle de tout un espace. Elle "dit adieu à toute l'école et à ses alentours" (52). Sa mère l'accueille à Tizi-Hibel pendant sept mois avant de la voir repartir, cette fois-ci, vers une nouvelle destination qui l'engagera sur une nouvelle voie. Son instruction lui permet d'être recrutée par l'hôpital chrétien des Aïth Manegueleth où s'accomplira sa destinée. Elle y reçoit son nom chrétien de Marguerite ainsi que son baptême et se marie avec un kabyle converti, Belkacem-ou-Amrouche.

Elle se retrouve alors à Ighil-Ali, dans la partie basse de la Kabylie, où le nouveau couple pensait s'《 installer pour la vie!» (109). Le nouvel espace est tout à fait différent de celui, religieux, des Aïth Manegueleth et même de celui du village natal. La narratrice confie d'ailleurs qu'elle " trouvai[t] beaucoup de différence entre [son] village de Tizi-Hibel et IghilAli » (109). De Tizi-Hibel à Ighil-Ali, en passant par Ouadhias, Taddert-ou-Fella et Aïth Manegueleth, Fadhma Aïth Mansour Amrouche passe de la Haute-Kabylie à la BasseKabylie. Mais à Ighil-Ali, sa belle-famille sombre dans la décadence après que son beaupère, dépensier, ait pris les rênes des Amrouche à la mort de l'aïeul.

Les nombreux déplacements, qui l'arrachent d'un espace géographique pour la livrer à un autre, interviennent jusque-là à l'intérieur des frontières du pays. C'est à ces frontières que s'arrêtent les limites de la première partie d'un long itinéraire qui se prolongera dans l'espace tunisien : "c'est ainsi que nous quittâmes le pays» (135). Pour pouvoir travailler et subvenir aux besoins du couple, Belkacem Ou-Amrouche est donc poussé à s'exiler, d'abord à l'intérieur du pays, à Souk Ahras, avant de traverser les frontières algériennes et s'établir à Tunis. Il est encouragé par sa femme qui a dû alors le rejoindre quelques mois plus tard avec ses trois enfants. «Il faut partir! Il faut que tu ailles chercher une situation avant que nous ne soyons sans abri " (132) l'engage-t-elle.

D'une maison à une autre, les déménagements se suivent dans Tunis et sont ponctués par des retours occasionnels au pays natal. Installée d'abord dans une maison arabe, la petite famille Amrouche déménage vers un quartier italo-sicilien, puis à la rue Chaker. Après la mort de son petit enfant, il faut partir. Déménager encore, pour s'installer 
dans "une grande chambre» (143) qu'il faut quitter au bout de deux mois pour un logement à l'impasse de l'Éventail qui, finalement, n'arrivera plus à contenir une famille qui a grandi. Il faut alors déménager de nouveau : "rue des Marchands d'huile ", "rue Abba», quartier de Bab-Aléoua,... C'est au bout du quatrième déménagement qu'intervient le premier retour au pays natal le temps des vacances. Puis un deuxième pour des "dernières vacances [...] dans la maison ancestrale» d'Ighil-Ali (152), et un troisième qui leur ouvre la porte de la nouvelle maison qu'on a fait à moitié construire sur un terrain cédé par les Pères. Puis, «le 2 août, la guerre éclata, et [Fadhma] du[t] passer l'année au pays, car Belkacem pouvait être appelé sous les drapeaux» (160). Elle repart à Ighil-Ali pour la quatrième fois où elle restera une année complète. Lorsqu'elle rentre à Tunis c'est encore pour s'installer dans une autre maison, rue du Fossé. Mais elle doit faire un dixième déménagement pour une dernière location, vers une maison arabe louée toujours à Bab-Aléoua. Une "relation conflictuelle» semble lier la narratrice à l'espace qui l'entoure ou qui l'accueille et qui lui impose l'exigence de partir pour d'autres espaces.

Le $1^{\text {er }}$ novembre 1918, la petite famille s'installe enfin dans sa propre maison, achetée à la rue de la Rivière où elle a vécu sept ans. Mais, il faudra encore, et pour la douzième fois, déménager parce que la maison de la rue de la Rivière est vendue et une autre est acquise dans la ville de Radès, "à dix kilomètres de Tunis» (199), où Fadhma Aïth Mansour a écrit, en août 1946, son histoire, qu'elle a complétée par un épilogue à Paris en juin 1962. Entretemps, un sixième retour au pays natal est organisé pour le mariage de Paul, le fils ainé. Le huitième retour à Ighil-Ali intervient après une longue absence de cinq ans. Le dixième, quant à lui, n'aura lieu, pour un court séjour, qu' « après huit ans d'absence » (194).

Bénéficiant d'un "passage gratuit pour la France» (188), et en compagnie de sa petite famille, Fadhma Amrouche foule pour la première fois le sol français, où elle est reçue par ses deux fils Henri et Paul. Ce premier voyage inaugure un itinéraire circulaire, Tunisie-France-Algérie-Tunisie, après s'être jusque-là limité aux allers-retours entre la Kabylie et Tunis. Des voyages à destination de l'hexagone qui en appelleront d'autres.

En Tunisie, l'installation à Radès n'est pas définitive. La maison finit par se vider, «les enfants étaient partis, les uns morts, les autres en France » (200). On lui trouve un acquéreur, on quitte définitivement la Tunisie et le retour pour le pays kabyle est voulu définitif.

Lorsqu'éclate la guerre d'Algérie, «les Pères Blancs déclarèrent que les ménages chrétiens devaient quitter leur demeure» (201). En 1956, la narratrice et son mari fuient, dans la foulée et l'affolement, leur propre pays natal comme des étrangers pour se réfugier à Paris. Ce vol pour la France à partir du pays natal inaugure un nouveau sens dans le long itinéraire d'exil. Mais le temps d'être rassuré, "malgré l'appréhension [des] enfants » (202), le couple Amrouche revient, obstinément, à Ighil-Ali pour la douzième fois, qui s'avèrera être, cette fois-ci, la dernière. Ils reviennent pour y finir leurs jours. " En posant les pieds sur le sol Algérie, [Fadhma] di[t]:» Adieu la France!» (203). Mais l'adieu sera finalement fait au pays natal. Après la mort de Belkacem, en 1959, son « compagnon de soixante années » (206), vieux et épuisé, la narratrice part pour la France, quittant à jamais Ighil-Ali et tout l'espace de son pays d'origine. Elle rejoint d'abord son fils René à Nice puis son autre fils Jean et sa fille Taos à Paris.

21 Voici donc retracé l'itinéraire de la narratrice fait de déplacements répétés et déchirants. Les espaces se relaient pour accueillir un personnage errant d'un village à un autre, d'une maison à une autre et d'un pays à un autre. Un itinéraire rythmé par 
des va-et-vient et des exils qui définissent deux espaces, deux frontières : l'ici et l'ailleurs

\section{L'ici et l'ailleurs : des frontières brouillées}

Tout exil se définit par rapport à un point de départ qui est le lieu de naissance. L'espace natal de la narratrice désigne les premières frontières géographiques de l'ici, à l'intérieur desquelles se rassemblent, sous la même couverture identitaire, les membres de la même communauté.

Le récit est ancré dans l'espace kabyle même à travers une écriture ethnographique qui témoigne d'un patrimoine culturel, architectural et traditionnel. Il est question de conte, de proverbes, d'us et de coutumes, et de rites. L'espace devient alors ethnique et plonge ses racines dans la terre natale. L'ici physique c'est Tizi-Hibel et Ighil-Ali en particulier, puis la Kabylie, et d'une façon générale l'Algérie.

24 Au-delà de l'espace physique, l'ici c'est aussi l'espace de la communauté musulmane kabyle dont a fait partie la mère de la narratrice, Aïni, une femme «très pieuse » (45). Fadhma Amrouche, qui est née musulmane, a fait entièrement partie de cet espace jusqu'à ses premières années d'enfance avant d'être engagée sur la voie chrétienne. Elle l'a été aussi partiellement même après son entrée dans le monde chrétien et ce en gardant des référents musulmans.

5 L'ailleurs, c'est par contre tout ce qui se situe donc au-delà des frontières, physiques ou non, de l'ici, du pays natal. C'est essentiellement Tunis et Paris. Le besoin de cet ailleurs est motivé par la nécessité de partir pour fuir une situation oppressante dans l'ici.

, ces lignes de démarcation entre l'ici et l'ailleurs sont repoussées, reconsidérées par le glissement identitaire de la narratrice en devenant chrétienne. De ce fait, une partie de cet ailleurs, où se trouve le français, le chrétien, soit l'espace qui n'était pas le sien initialement, se transforme en un ici dans lequel elle se reconnait partiellement. Lorsque enfant, Fadhma Aith Mansour était encore musulmane, à son ici de l'enfance s'oppose l'ailleurs qui se situe chez la communauté chrétienne dans la Kabylie même. Cet enchevêtrement spatial et identitaire dessine l'image d'un ici et d'un ailleurs qui s'entremêlent.

27 Cependant, l'ici semble n'être nulle part du fait du double rejet que la narratrice a subi de la part des siens et des Français. Pour les Kabyles, elle et sa petite famille sont des «Roumis, des renégats» (203). Et ce rejet est essentiellement nourri par la différence religieuse. Pour les Français, ils sont, par contre, «des bicots comme les autres» (203). À l'hôpital des Aïth Manegueleth, par exemple, on «ne désirait pas que les Kabyles sortent de leur milieu» (96). Pour le mariage de Fadhma Amrouche, la Mère Supérieure ne consentait pas à lui offrir "des souliers européens" mais juste «des chaussures en cuir rouge, à la mode indigène, appelées "thirihith" » (203).

Du fait de ce double rejet, Tizi-Hibel, et le pays kabyle d'une façon générale, cesse d'être l'ici qu'il est censé être entièrement. Il devient le point de départ des exils de Fadhma Amrouche, le témoin de ses déplacements et parfois même la cause. Cette opposition entre l'ici et l'ailleurs trouve son prolongement dans la dualité entre le même et l'autre.

Dans son ensemble, le cadre spatial de Histoire de ma vie se présente en deux grandes parties. L'histoire se déroule dans l'Algérie colonisée où s'affrontent deux espaces: l'Algérie vs la France, dominé vs dominant, colonisé vs colonisateur, autochtone vs 
colon, terre d'origine vs terre d'accueil. Ces oppositions, qui définissent l'ici et l'ailleurs, peuvent se résumer à l'opposition globale qui met face à face le même et l'autre. Et cette dualité est déterminée par l'élément prépondérant qu'est la religion pour donner lieu à son tour à l'opposition musulman vs chrétien autour de laquelle est tissée l'essentiel de la trame narrative qui rend compte d'un monde à deux pôles.

Du côté de l'espace natal se trouve donc le même, qui est l'Algérien, l'autochtone, le dominé, le colonisé. Fadhma Aïth Mansour Amrouche est née Algérienne, a été autochtone par son enracinement, et dominée du fait de la colonisation du peuple dont elle a fait partie. C'est tout cela qu'elle a en commun avec les siens.

Les siens sont ceux qui forment toute cette communauté kabyle avec lesquels elle partage une culture, une langue, des traditions,... soit tout un espace natal. Mais elle n'est pas musulmane comme la majorité des «siens». Elle s'en détache du fait de sa religion chrétienne. Et cet élément distinctif de la religion qui la différencie d'eux tronque ainsi le sens du même. Dans quel même se reconnaît donc Fadhma Amrouche, le Kabyle ou le Français? Tout en l'éloignant des siens, sa religion est censée la rapprocher du Français qui cesse de ce fait d'être vraiment l'autre. Ceci nous amène à nous interroger sur le sens aussi de l'autre chez la narratrice. Qui est l'autre, le français ou le kabyle musulman?

Est-ce qu'il faut considérer le même du point de vue des origines, du sol, ou de celui de la religion?

Au même temps qu'ils s'opposent, le même et l'autre sont deux espaces ambivalents avec des frontières brouillées, à l'image de l'ici qui s'entremêle avec l'ailleurs.

La narratrice se trouve, par conséquent, livrée à des espaces de vie qui ne l'accueillent pas dans les mêmes conditions selon qu'ils soient ouverts ou clos.

\section{Espaces clos/espaces ouverts : dysphorie de l'enfermement et euphorie du dehors}

La relation conflictuelle avec l'espace fait naître chez Fadhma Aïth Mansour la nécessité de partir à la recherche d'une terre clémente. Chacun de ces nombreux espaces l'accueille dans des conditions différentes. Par-ci, ce sont des conflits, des animosités qui naissent dans des espaces oppresseurs, imposant des départs, par-là s'exprime par contre un sentiment de bien-être et de symbiose avec l'espace accueillant.

La dysphorie de l'enfermement s'exprime dans les espaces diégétiques clos que sont une école, un hôpital et surtout des maisons. La première maison introduite par la narration est celle de la mère Aïni, qu'a investie son grand frère venu lui demander de la quitter. Elle est le premier motif de discorde qui appellera un conflit et bouleversera toute la vie de la mère. C'est dans ce même espace, théâtre d'une relation extraconjugale, que celle-ci a accouché, " toute seule, avec ses deux petits; personne auprès d'elle pour l'assister " (25). Cette maison, objet aussi d'un bras de fer avec les beauxfrères, n'a pas connu de moments de joie, mais plutôt de douloureuses séparations. C'est celle que quittera un jour le fils Lâmara. » Tu vas partir dans ta maison, car il s'y est passé quelque chose » (68) lui dit la femme du cheikh.

36 Fadhma Aïth Mansour a sûrement connu des moments de bonheur dans la maison maternelle, ne serait-ce que du fait de la compagnie de sa mère. Mais force est de constater que la narration ne rend compte d'aucun enthousiasme d'y vivre : «j'ai passé 
ces vacances-là comme toutes les autres à la maison " (42). La maison est présentée tout juste comme un abri et un lieu où sont convoqués des souvenirs d'enfance. Pour la narratrice, c'est malgré elle qu'elle retourne vivre "dans cet espace restreint» (58), lorsque l'école de Taddert-ou-Fella est dissoute. D'une façon globale, elle ne semble pas s'épanouir dans ces espaces fermés. À l'hôpital des Aïth Manegueleth, elle n'est pas logée à meilleure enseigne, bien que ce soit dans cet espace que sa destinée s'est accomplie. Mais ce n'est pas cela qui est mis en relief, plutôt des souvenirs qu' « un jour, un homme mourut dans la salle» (76) ou de "la promiscuité des malades avec les bien portantes» (74).

À l'intérieur de ces espaces fermés, la description porte, à l'aide de prédicats négatifs, sur une vie de malaise et de mal-être moral et ce depuis la maison de la mère, jusqu'à la demeure des beaux-parents à Ighil-Ali où " la pièce était immense, dix mètres de long, au moins, sur sept ou huit de large [...]. Aucun confort ni aucune propreté» (91).

À Tunis, également, les déménagements se répètent en quête du bien-être et d'espaces ouverts sur le monde. L'enfermement des lieux corrompt l'atmosphère de l'intérieur et génère l'étouffement. Voir le dehors et le monde paraît comme une nécessité vitale qui pousse la narratrice vers la moindre ouverture. Dehors, Fadhma Amrouche semble se libérer de ses angoisses, et de ses appréhensions qu'engendre l'intérieur des maisons.

Le récit de Histoire de ma vie s'ouvre sur un cadre spatial ouvert, celui d' "un endroit écarté, en dehors du village, appelé" sebala ", où tous les villageois dressent leurs meules » (23). Il est suivi par un espace fermé qui est celui de la maison de la mère avec sa cour commune. Mais cette cour est un espace mi-ouvert, mi-clos, puisque tout en étant ouverte à tous les occupants des maisons qui l'entourent, il reste fermé à toute personne qui lui est étrangère. La cour commune ne diffère pas beaucoup de tajmaât, espace public qui trône au milieu de chaque village kabyle. Lieu de réunion exclusivement masculin, tajmaât est l'espace de la préservation de la tradition et de l'honneur, de la prise d'engagement et de décisions graves. Si tajmaât est un espace ouvert, il ne l'est cependant que pour les hommes. Les femmes, pour lesquelles il est fermé, en sont exclues. Tout comme la cour commune, mi-ouverte, mi-close, tajmaât est, à ce propos, aux frontières des deux espaces.

Le récit de Histoire de ma vie fait place à ce genre de lieux mais évolue essentiellement dans des espaces qui restent entièrement ouverts à l'exemple des champs qui s'ouvrent, sans frontières, à tous. La narration leur réserve une place de choix. À Taddert-ou-Fella, Fadhma Aïth Mansour Amrouche a eu à vivre "plus souvent dehors que dedans» (35). Elle respire l'euphorie du dehors qu'elle trouve dans l'infinité des espaces ouverts comme les champs, espace nourricier, de récoltes, de rencontres, de travail et des libertés: "Nous avions un champ de figuiers, qu'on appelait Thoujal. C'était là que j'aimais me rendre avec ma mère» (59).

41 La nature est gratifiée d'adjectifs valorisants et décrite à l'aide de prédicats positifs. La narratrice s'épanouit dans cette nature qui la soulage comme d'un joug:

J'ai gardé un souvenir lumineux de ces promenades, les soirs de printemps ou d'été. Je reverrai toujours les arbres d'églantines et de clématites dont nous faisions des guirlandes, et les chèvrefeuilles odorants, les tapis de marguerites jaunes et blanches, et les bleuets, et les boutons d'or. Je n'ai jamais vu depuis autant de fleurs, ni pareil paysage (33). dont le « cœur [est] assoiffé d'affection» (81). 
deux espaces, clos et ouverts, qui s'opposent révèlent l'état d'âme changeant de la narratrice, entre euphorie et dysphorie. Soit deux états d'âme contradictoires qui renvoient à un espace mental qui transcende les frontières physiques comme le fait aussi l'espace mnémonique. Le récit est dicté par une mémoire partagée qui fait des vaet-vient entre le présent et le passé.

\section{Espace mnémonique : souvenirs vs oublis, présent vs passé}

Le type de formes de durée narrative le plus présent dans Histoire de ma vie est l'ellipse qui passe sous silence des pans entiers de l'histoire racontée. La narratrice assume parfois ces élisions en indiquant sa volonté de faire l'impasse sur des étapes de sa vie : "de toutes les filles qui sont passées par l'école, je ne dirai rien, je n'ai pas grand-chose à en dire» (33). Dans d'autres cas, l'élision n'est ni explicite, ni déterminée. On ne saura rien des événements qui ont pu marquer les années 1938, de 1942 à 1945, de 1947 à 1952 et de 1960 à 1962. Dans le cas d'un (e) auteur (e) écrivant son autobiographie au crépuscule de son âge, l'ellipse "temporelle " peut être le fait d'un choix auctorial à croire certains passages narratifs dans notre corpus : « de ma vie à Radès, je ne parlerai pas beaucoup, car tous mes enfants la connaissent. Je me bornerai à rappeler quelques souvenirs" (183). L'élision ne touche pas ici tout un segment temporel mais une partie de celui-ci. Elle est alors partielle.

s derrière ce choix assumé n'y a-t-il pas une part d'oublis qui empêchent la narratrice de tout raconter? De nombreux exemples de récits elliptiques disent explicitement la difficulté de la mémoire de livrer les souvenirs de la longue vie d'une octogénaire. De la période passée aux Ouadhias, la narratrice ne garde comme souvenirs que des images: "je me souviens très peu de cette époque. Des images, seulement des images » (27). Le souvenir de son déplacement vers l'école de Taddert-ou-Fella n'est pas plus net: « de ce voyage je ne me souviens pas; je me rappelle seulement qu'en descendant à la rivière, nous avons mangé des arbouses » (30). Il en est de même des premières années de l'école de Taddert-ou-Fella: "quand je suis arrivée, j'étais encore bien jeune, et je me souviens peu des premières années de mon séjour à l'école » (32).

Les informations livrées par la mémoire de la narratrice deviennent incertaines concernant plusieurs faits lointains : «je ne sais pas au juste combien de jours j'ai passés à Mekla» (48). À propos de Mekla, la mémoire est incapable d'aller au-delà de certains souvenirs : "je n'ai guère d'autre souvenir " (49). À l'hôpital des Aïth Maneguelleth, elle n’a pas pu se souvenir des paroles du Père Supérieur : « il me posa plusieurs questions dont je ne me souviens plus bien, car j'étais occupée à détailler ses traits» (73). L'oubli est ici expliqué, pour une fois, par l'inattention. La mémoire devient défaillante même pour les étapes les plus importantes dans la vie de la narratrice, comme la journée de son baptême et la cérémonie de son mariage: "je ne me rappelle pas très bien comment les choses se sont passées ce matin-là » (87).

Le verbe intransitif "se souvenir ", marqué à chaque fois par les adverbes de négation, revient souvent pour traduire les oublis de la narration. C'est ce que nous pouvons confirmer avec cet autre exemple : «je ne me souviens pas si c'est en 1927 ou 28 que MarieLouise réussit au certificat d'études " (186). Ces souvenirs qui se placent entre tous ces oublis sont fragiles. Les verbes de l'incertitude auxquels fait recours la narratrice 
confirment cette fragilité : "c'était pendant l'hiver, pour les vacances du mois de janvier, je crois» (49). Et c'est souvent à l'aide du verbe "croire», utilisé dans le sens de "supposer que», que la narratrice signifie l'incertitude de certaines dates: «je crois que c'est le vendredi après mon mariage que je vis arriver Hemma» (88). Il est fait recours aussi à des adverbes, dont ceux signifiant le doute comme l'adverbe "peut-être»: "nous restâmes quelques semaines, peut-être un mois, à attendre » (50).

Malgré ces lacunes, la mémoire n'est pas qu'oublis, parce que des souvenirs s'y accrochent tantôt profusément, "beaucoup de choses oubliées me revinrent alors à la mémoire " (48), tantôt parcimonieusement: «un souvenir de l'école me revint » (62). Ils reviennent aussi avec force détails : «nous partîmes donc, ce samedi 26 août, en passant par l'hôpital » (89). Et ce type de précisions se répète dans le récit : « c'est le 7 août, un mardi, que mon mari quitta la maison paternelle pour l'inconnu » (132).

Incertitudes, oublis, souvenirs fragiles, souvenirs accrocheurs malgré tout, le récit de vie n'est qu'un vaste espace de la mémoire qui se construit entre le présent et le passé. Le souvenir et l'oubli se présentent comme deux éléments constitutifs de cette mémoire qui oublie, qui se rappelle, qui fléchit et qui se ressaisit pour que la narratrice "lègue ce dont [elle a] pu [se] souvenir» (199) pour sa fille Taos. Ce sont autant de déplacements qui s'opèrent dans un espace mnémonique ambivalent.

Pour conclure retenons que l'écriture de l'errance de Fadhma Aith Mansour Amrouche est construite dans et au delà des frontières de l'espace physique. Elle nous livre un espace, matériel ou mental, dans tous ses états. L'errance a livré la narratrice à deux espaces, deux frontières qui s'opposent : l'ici et l'ailleurs. Ces deux espaces s'entremêlent et il devient difficile de les distinguer d'autant que le double rejet de la narratrice, de la part des Kabyles et de ses coreligionnaires, fait que l'espace de l'ici n'est nulle part. Cette confusion nous a amené à nous interroger sur la distinction du même et de l'autre. Nous sommes donc face à deux espaces ambivalents aux frontières brouillées.

51 L'ambivalence spatiale se dégage aussi de l'opposition entre les espaces ouverts et les espaces clos. L'euphorie, suscitée par les premiers, tranche avec la dysphorie des seconds espaces, et les deux traduisent une ambivalence dans un espace mental à travers deux états d'âme contradictoires. Cette ambivalence, qui transcende les frontières matérielles, se reflète aussi dans l'espace mnémonique lorsque la mémoire fait des va-et-vient entre le présent et le passé. Elle livre des souvenirs et en oublie d'autres. Ainsi, ces déplacements entre le passé et le présent s'opèrent dans un espace mnémonique ambivalent.

Le récit qui nous est donné à lire est celui d'une octogénaire livrée à l'errance. Elle est étrangère partout, et apparaît comme un personnage "liminaire», que Marie Scarpa définit comme faisant partie de "ces figures bloquées sur les seuils, figées dans un entredeux constitutif et définitif, "inachevées" »(Scarpa, 2009 : 25).

Pour paraphraser Florence Paravy, nous remarquerons que « la relation avec l'espace natal est essentielle, mais conflictuelle, et [Fadhma] apparaît déjà comme étrang[ère] au monde qui l'entoure. Il faut donc le quitter, devenir un peu plus étrang[ère], dans un monde nouveau où le personnage se sent déplacé, coupé de lui-même » (Paravy, 1999 : 38).

54 L'ambivalence qui se dégage de tous ces espaces rejoint l'ambivalence identitaire du personnage de la narratrice. Figée dans un entre-deux, Fadhma Aïth Mansour 
Amrouche est déchirée entre deux cultures, deux identités distinctes, kabyle et française.

\section{BIBLIOGRAPHIE}

AITH MANSOUR AMROUCHE, Fadhma, Histoire de ma vie, Edition Mehdi, Algérie, 2009. 219 p.

Première édition Maspero, Paris, 1968. 199 p.

BONNET, Véronique, De l'exil à l'errance : écriture et quête d'appartenance dans la littérature contemporaine des petites Antilles anglophones et francophones [en ligne], thèse de doctorat, université Paris XIII, 1997, sous la direction de Charles Bonn et Jean-Louis Joubert. P.278. Disponible sur [www.limag.refer.org/Theses/Bonnet.PDF] (Consulté le 07 décembre 2014).

HACHETTE Livre, Paris, 1999.

PARAVY, Florence, L'espace dans le roman africain francophone contemporain (1970-1990),

L'Harmattan, Paris, 1999.

SCARPA, Marie, Le personnage liminaire, Romantisme, 2009/3 n 145, p. 25-35. [En ligne], disponible sur [www.cairn.info/revue-romantisme-2009-3-page-25.htm] (Consulté le 01 mars 2015).

\section{RÉSUMÉS}

Le récit de Histoire de ma vie, de Fadhma Aïth Mansour Amrouche, est construit dans et au-delà des frontières physiques. L'errance a livré la narratrice à deux espaces, l'ici et l'ailleurs, qui s'opposent et s'entremêlent. Il devient alors difficile de les distinguer, d'autant que, du fait de son double rejet, la narratrice est étrangère partout et l'ici est nulle part. Les frontières sont brouillées et il est difficile de différencier le même de l'autre. L'ambivalence spatiale reflète l'ambivalence identitaire du personnage, déchiré entre deux cultures distinctes.

The narrative of Histoire de ma vie, of Fadhma Aith Mansour Amrouche, is built in and beyond physical borders. Wandering has delivered the narrator to two spaces, the here and the elsewhere, which oppose and intermingle. It then becomes difficult to distinguish them, especially, because of her double rejection, the narrator is foreign everywhere and the Here is nowhere. Borders are blurred and it is difficult to differentiate between the same and the other. The spatial ambivalence reflects the identity ambivalence of the character, torn between two distinct cultures.

\section{INDEX}

Mots-clés : ambivalence, espace, frontières, ici/ailleurs, même/autre

$$
\text { الازدواجية, الفضاء, الحدود, هنا/ و هناك, التشابه/ الاختلاففهرس الكلمات المفتاحية: }
$$

Keywords : ambivalence, space, borders, wandering, here/elsewhere, same/other 
AUTEUR

KAMEL MEDJDOUB

Université d'Alger 2 